\title{
INFERENTIAL-LEARNING CONTROL OF QUALITY PROPERTIES IN SEMI-BATCH REACTORS
}

\author{
Jesus Flores-Cerrillo and John F. MacGregor \\ Department of Chemical Engineering, McMaster University, \\ Hamilton, Ontario, Canada, L8S4L7
}

\begin{abstract}
A strategy for controlling product quality properties, subject to batch-to-batch and within batch disturbances is presented. The methodology is based on readily available measurements, information extracted from existing databases and a few simple identification experiments. This approach extends the mid-course correction control strategies to overcome model error when the process is affected by constant, correlated and uncorrelated disturbances. Adaptive PLS (Partial Least Squares) methods are used to extract the information and to update the models used for prediction and control. The methodology is illustrated with a few emulsion polymerisation examples. Copyright (C) 2002 IFAC
\end{abstract}

Keywords: Batch control, Learning control, Partial Least Squares, Statistical process control, Polymerisation, Particle Size Distribution.

\section{INTRODUCTION}

Batch/semibatch processes are commonly used to produce speciality chemicals, polymers and pharmaceuticals. In these processes, it is necessary to follow target quality trajectories and achieve tight final quality specifications. However, quality tracking is not easily achieved because processes are subject to variations in raw material properties and errors in initialisation. Moreover, disturbance compensation is difficult due to the non-linear behaviour of the properties and to the fact that robust on-line sensors for quality variable monitoring are rarely available.

Large errors in initialisation and sequencing can be minimized through high degree of automation and feedback control of easily measured variables such as temperature, level and pressure. However, raw material variations, and process condition changes still may affect the quality trajectories and final product specifications.

Several approaches have been presented to control quality properties in batch processes. However, most of them are based on complex theoretical models and computationally intensive control strategies (Crowley, et al., 2000; Kozub, 1989). These strategies are difficult to implement in industrial settings because they require frequent and instantaneous on-line measurements as well as perfect model knowledge. On the other hand, empirical modelling has the advantage of using information routinely collected and easiness in model building (Flores-Cerrillo and MacGregor, 2002; Russell, et al., 1998). However, model error arising from low quality dataset or changing disturbances and process conditions may degrade model prediction and control.

One way to reduce model error is by using past control errors in the incoming process; this is known as Iterative Learning Control (ILC) or batch-to-batch control (BBC) and has been recently used in the chemical community (Lee, et al., 2000). Under this perspective, a few authors have combined ILC with on-line control algorithms to reject correlated and uncorrelated disturbances. These approaches have been successful at some extent. However, they require large experimentation for model identification (Lee, et al., 2001). 
The purpose of this study is to introduce a methodology that uses poor databases, easily obtained on-line process measurements and a few off-line delayed analysis to control batch and semibatch quality properties and trajectories when the process is subject to error initialisation, constant, correlated and uncorrelated disturbances. The contents of this paper are as follow: in section 2 the adaptive-learning mid-course correction methodology is introduced; in section 3 , the methodology is illustrated with emulsion polymerisation case studies. In section 4, conclusions and future work are drawn.

\section{ADAPTIVE-LEARNING CONTROL METHODOLOGY}

The proposed Methodology extends the Mid-course Correction (MCC) strategy (Yabuki and MacGregor, 1997) to overcome model error and to control quality trajectory property. Model error and process changing conditions are overcome using adaptivelearning schemes to take into account new information and the repetitive nature of the disturbances.

In the MCC strategy, a mid-course corrective action is taken whenever the predicted final properties fall outside a defined control region. The prediction is done using a PLS model built from historical data. PLS regression technique is employed because in process data, high correlation among variables leads to ill-conditioned parameter estimation, and generally exist larger number of variables than observations. PLS overcome these problems by projecting the original variables onto lower dimensional subspaces:

$$
\begin{aligned}
& \mathbf{X}=\mathbf{T} \mathbf{P}^{\mathbf{T}}+\mathbf{E} \\
& \mathbf{Y}=\hat{\mathbf{U}} \mathbf{Q}^{\mathbf{T}}+\mathbf{F}
\end{aligned}
$$

where $\mathbf{T}$ and $\hat{\mathbf{U}}$ are new latent variables that captures most of the data variability, reducing the variance of the parameter estimates at expenses of a small bias (see Geladi, et al., 1986 for details).

For control purposes, the PLS model is build by including in the $\mathbf{X}$ matrix, on-line $\left(\boldsymbol{x}_{\boldsymbol{o n}}\right)$, off-line $\left(\boldsymbol{x}_{\boldsymbol{o f f}}\right)$ process measurements and control actions $\left(\boldsymbol{x}_{\boldsymbol{c}}\right)$ (nominal model):

$$
\begin{gathered}
\hat{\mathbf{y}}=f\left(\mathbf{X}_{\mathbf{m}}, \mathbf{X}_{\mathbf{c}}\right)=\hat{\beta} \mathbf{X} \\
\mathbf{X}_{\mathbf{m}}=\left[\mathbf{X}_{\text {on }} \mathbf{X}_{\text {off }}\right]
\end{gathered}
$$

where $\hat{\mathbf{y}}$ are the predicted quality properties.

When a new batch is being produced, process measurements $\mathbf{x}_{\mathbf{m}}$ are collected, and a prediction is performed:

$$
\begin{aligned}
\hat{\mathbf{y}} & =\hat{\beta} \mathbf{x} ; \quad \mathbf{x}_{\mathbf{C}}=\mathbf{x}_{\text {sp }} \\
\mathbf{x} & =\left[\begin{array}{lll}
\mathbf{x}_{\text {on }} & \mathbf{x}_{\text {off }} & \mathbf{x}_{\mathbf{C}}
\end{array}\right]
\end{aligned}
$$

where $\mathbf{x}_{\mathbf{s p}}$ are the set-points of the manipulated variables.
If the prediction $\hat{\mathbf{y}}$ is outside a statistical defined control region ( $\mathrm{T}^{2}$ Hostelling), it is necessary to perform control action to track on target the quality properties. This is accomplished by inverting (2) according to:

$$
\begin{gathered}
\underset{\mathbf{x}_{\mathbf{c}}}{\min }\left\|\mathbf{y}_{\mathbf{s p}}-\hat{\mathbf{y}}\left(\mathbf{x}_{\mathbf{c}}\right)\right\|^{2} \text { such that } \\
\mathbf{x}_{\mathbf{c}} \geq 0
\end{gathered}
$$

The $\mathrm{T}^{2}$ Hostelling is computed by performing principal component analysis (PCA) on the quality variables when the process is subject only to normal variation:

$$
\mathbf{X}=\mathbf{T} \mathbf{P}^{\mathbf{T}}+\mathbf{E}
$$

Mid-course correction strategy is developed for controlling final properties. However, it is straightforward to control full trajectories by simply dividing these trajectories into a few intervals and performing mid-course correction on each one of them. It worth to point out some of the possible alternatives for control: 1) Build a PLS model for each interval having as control region the end of such interval; 2) Build a PLS model for each interval but having as control region the end of the batch, and 3) Build a single PLS model using as control region the end of the batch (Russell, et al., 1998). Trajectory segmentation and control action is illustrated in Figure 1.

No matter what of the PLS control strategies is chosen, it is generally assumed (Russell, et al., 1998; Yabuki and MacGregor, 1997; Flores-Cerrillo and MacGregor, 2002) that the training data set contains sufficient input movements and disturbance information to allow proper model identification; i.e. building of almost a perfect model and no change in disturbance type. This situation is not always possible, especially if the model is built from historical data. Model error leads to incorrect control action and then to undesired product qualities. To overcome this deficiency, an adaptive-learning midcourse correction strategy is introduced. The idea is to update model parameters, in every interval, at the end of the batch by combining the new batch information with the previous data set. Several adaptive learning algorithms have been studied; however, for clarity in the exposition only two are presented.

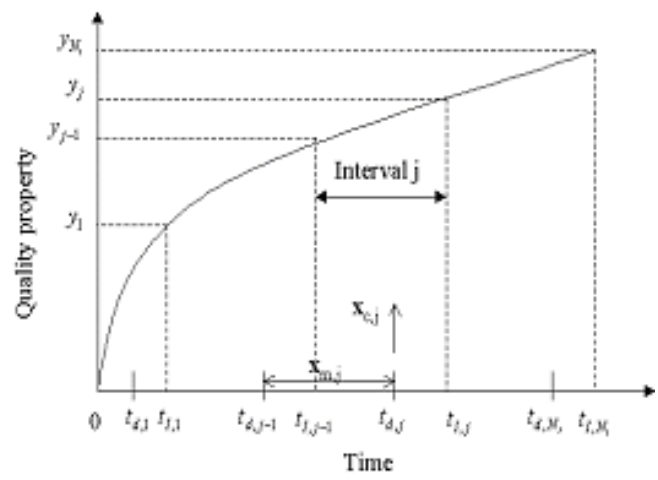

Fig. 1. Trajectory segmentation and control action. 
Adaptive model: This approach updates the PLS model at the end of the batch:

$$
\mathbf{Y}^{(0)}=\mathbf{Y}_{\mathrm{tr}} ; \quad \mathbf{X}^{(\mathbf{0})}=\mathbf{X}_{\mathrm{tr}} ; \quad \mathbf{y}^{(\mathbf{0})}=\hat{\boldsymbol{\beta}}^{(\mathbf{0})} \mathbf{X}^{(\mathbf{0})}
$$

for a batch $k$

$$
\begin{aligned}
& \mathbf{Y}^{(\mathbf{k})}=\left[\begin{array}{l}
\mathbf{Y}^{(\mathbf{k}-\mathbf{1})} \\
\mathbf{y}^{(\text {new })}
\end{array}\right] ; \quad \mathbf{X}^{(\mathbf{k})}=\left[\begin{array}{l}
\mathbf{X}^{(\mathbf{k}-\mathbf{1})} \\
\mathbf{X}^{(\text {new })}
\end{array}\right] \\
& {\left[\mathbf{Y}^{(\mathbf{k})} \mid \mathbf{X}^{(\mathbf{k})}\right] \stackrel{P L S}{\longrightarrow} \hat{\boldsymbol{\beta}}^{(\mathbf{k})}}
\end{aligned}
$$

update model is :

$$
\mathbf{y}^{(\mathbf{k})}=\hat{\boldsymbol{\beta}}^{(\mathbf{k})} \mathbf{x}^{(\mathbf{k})}
$$

where $(\mathrm{o})$ indicates original matrices, and tr training data set.

Adaptive+Prediction error (PE) model: This approach first updates model parameters (6) followed by addition of a prediction error term:

$$
\text { update model is : }
$$

$$
\begin{aligned}
& \mathbf{y}^{(\mathbf{k})}=\hat{\boldsymbol{\beta}}^{(\mathbf{k})} \mathbf{x}^{(\mathbf{k})} \\
& \hat{\mathbf{e}}^{(\mathbf{k})}=\hat{\boldsymbol{\alpha}}^{(\mathbf{k})} \mathbf{x}_{\mathbf{c}}^{(\mathbf{k})} \\
& \mathbf{y}^{(\mathbf{k})}=\hat{\boldsymbol{\beta}}^{(\mathbf{k})} \mathbf{x}^{(\mathbf{k})}+\hat{\mathbf{e}}^{(\mathbf{k})}
\end{aligned}
$$

The adaptive methodology is schematically illustrated in Figure 2.

\section{SIMULATION STUDIES}

To illustrate the main points of the methodology, two emulsion polymerisation case studies are presented: Case study I for controlling trajectories of number of particles (Np) and Conversion (X) in batch and semibatch process, and Case study II for controlling particle size distribution (PSD) in batch reactors. Emulsion polymerisation was selected because control of the latex quality specifications is not easily achieved due to the quality properties are extremely sensitivity to small changes in impurity concentrations, surfactant properties, feed flow rate and temperature. These studies try to resemble industrial process conditions in which only easily available on-line and a few off-line measurements are used.

\subsection{Case Study I. Control of Np and X}

A non-linear model to simulate styrene emulsion polymerisation was developed by Lynch and Kiparissides (1981), and used in this case study for data generation and model performance evaluation. This model has been lightly modified for use in both batch and semi-batch policies. For a complete description of the model, model parameters, process conditions and recipes the reader is referred to the original publication.

Disturbances, manipulated and controlled variables. In emulsion polymerisation systems the major disturbances affecting the quality trajectories of number of particles $(\mathrm{Np})$ and conversion $(\mathrm{X})$ are the emulsifier particle size coverage $\left(a_{\mathrm{s}}\right)$ and initiator decomposition efficiency $(f)$. In the following examples we restrict control actions to shots of emulsifier and monomer because they have fast dynamics on the controlled variables. Emulsifier shots will be performed when the process is affected by negative disturbances (low $a_{s}$ and $f$ ) while shots of monomer will be introduced when raw characteristic materials are above normal conditions $\left(a_{s}=5.62 \times 10^{-5}\right.$ and $f=0.6$ ).

\subsubsection{Batch emulsion polymerisation example.}

In this example, the control action is a shot of emulsifier and/or monomer at 55min (total reaction time $480 \mathrm{~min}$ ). In order to predict $\mathrm{Np}$ and $\mathrm{X}$, off-line measurements (sampled at 40min) in $\mathrm{X}$ and particle diameter $(\mathrm{Dp})$ together with on-line measurements in the jacket $(\mathrm{Tj})$ and reactor temperature $(\mathrm{Tr})$ are considered available. The training data set has the normal random distributed measurement errors reported in Table 1 .

Data Generation: A PLS model was built from a data set consisting of 13 batch observations in which $a_{s}$ and $f$ were randomly varied: 11 under normal operating conditions and two in which mid-course correction was performed. This model has large parameter mismatch and is used as nominal model (eq. 2, section 2) from which the learning control algorithms (eq. 6-7) are compared.

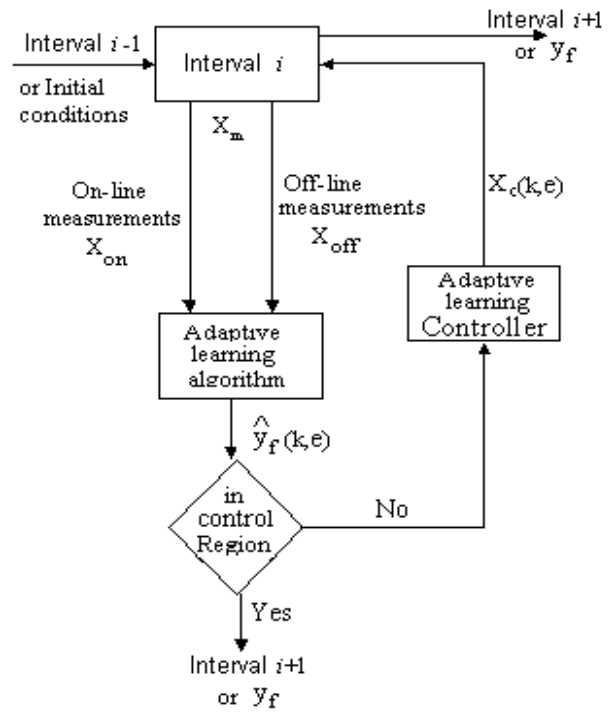

Fig. 2. Adaptive mid-course correction Methodology.

Table 1. Measurement noise and sampling

\begin{tabular}{cccc} 
& \multicolumn{2}{c}{ Noise } & \multicolumn{2}{c}{ Sampling time (min) } \\
Measurements & $\sigma \%$ & Batch & Semi-batch \\
\hline $\mathrm{Tj}^{\mathrm{a}}$ & 0.1 & $10 \mathrm{~min}$ & Every 10min \\
$\mathrm{Tr}^{\mathrm{a}}$ & 0.1 & $10 \mathrm{~min}$ & Every 10min \\
$\mathrm{X}^{\mathrm{b}}$ & 0.5 & 40,480 & $40,110,185,300,480$ \\
$\mathrm{Dp}^{\mathrm{a}}$ & 0.576 & 40,480 & $40,110,185,300,480$ \\
$\mathrm{~Np}^{\mathrm{b}}$ & 1.73 & 480 & 480
\end{tabular}

${ }^{\mathrm{a}}$ Flores-Cerrillo and MacGregor, (2002), ${ }^{\mathrm{b}}$ assumed 
To illustrate control performance some results are presented. In Figure 3, is shown the evolution of the final quality properties (scaled units) for the case in which a constant time-invariant negative disturbance affects the process $\left(\mathrm{T}^{2}\right.$ Hostelling ellipse is obtained from the normal variations in $a_{s}=1.5 \%$ and $f=3 \%$ based on one standard deviation). In Figure 4 is shown the evolution of the controlled variables for a correlated disturbance (disturbance repeats every 5 batches). In these Figures (.) represents control using nominal model; (.-) the adaptive PLS model; and (+-) the adaptive-prediction error model. It can be observed that the non-learning model cannot track on target bad batches, while the adaptive schemes are successful (these results are typical from others obtained using different types and magnitudes of disturbances). The results of these two adaptive methods are quite similar in most of the cases, indicating that any of them can be used.

\section{Control Results}

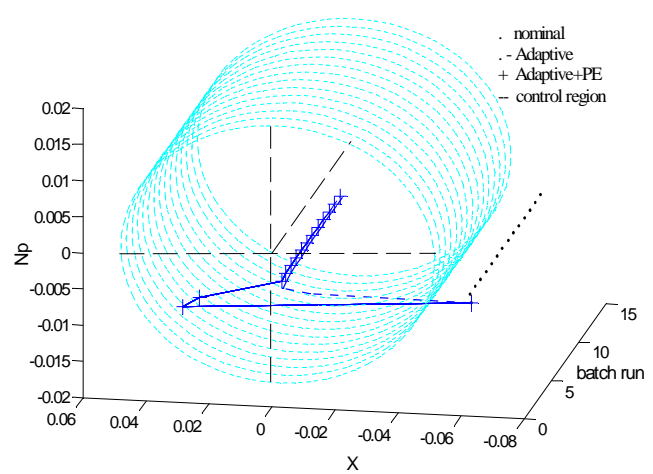

Fig. 3. Control for constant disturbance. $a_{s}=5.06 \times 10^{-5}$ and $f=0.54$.
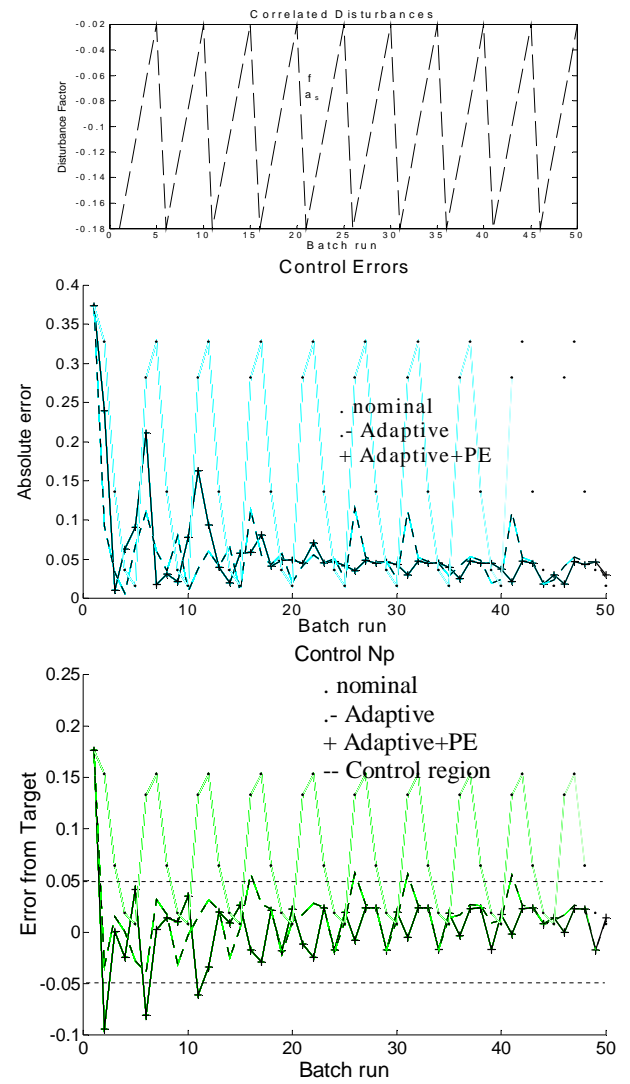

Fig. 4. Control for correlated disturbance.

\subsubsection{Semi-batch polymerisation example}

In this example, $\mathrm{X}$ and $\mathrm{Np}$ trajectories in a semibatch processes are tracked on target. At difference from the batch studies, in semi-batch process, emulsifier and monomer are continuously added to the system. Therefore, disturbance of emulsifier $a_{\mathrm{s}}$ is time-variant.

The Quality trajectories are divided in 4 intervals, every one with an end control quality target-region $(\mathrm{X}, \mathrm{Np})$. On-line $(\mathrm{Tj}, \mathrm{Tr})$ and off-line process measurements (X, Dp with analysis delay of $15 \mathrm{~min}$ ) corrupted with noise (Table 1) are used. The characteristics of these segments are reported in Table 2. A nominal PLS model, for each segment trajectory, was built from a training set consisting of 13 observations: 9 under normal raw material variations in $a_{s}$ and $f$, and 4 in which some MCC were performed.

In Figure 5a and $5 \mathrm{~b}$ is shown the performance of the adaptive control algorithm in tracking the trajectories of $\mathrm{X}$ and $\mathrm{Np}$ when constant disturbances (batch 1-10, $a_{s}$ and $f$ decrease by a factor of 0.9 and from batch $11-20$ by a factor 0.85 ) and initialisation errors in emulsifier, monomer and initiator affects the process. In these figures (-) represent the target, and (x--, $\left.*_{--}\right)$ the tracked trajectories after 9 and 20 batches respectively. Run number 1 does not track on target the trajectories due to large model mismatch. However, after few runs, the trajectories are tracked on target; in batch 10 a higher disturbance affect the processes. However, the control performance is not decremented; after 20 batches, the tracking is satisfactory. Finally, it should be mention that this example is with illustrative purposes, since to achieve such tight control trajectory in $\mathrm{Np}$, it is necessary to promote new nucleations, situation that may lead to high emulsifier concentration at the end of the process.

$\underline{\text { Table 2. Trajectory segmentation in semi-batch case. }}$

\begin{tabular}{cccc} 
& \multicolumn{2}{c}{ Time $(\mathrm{min})$} & \\
Segment & Measurements & Control & Targets \\
\hline 1 & 40 & $50-55$ & 75 \\
2 & 110 & $125-140$ & 160 \\
3 & 185 & $200-215$ & 275 \\
4 & 300 & $315-325$ & 480
\end{tabular}

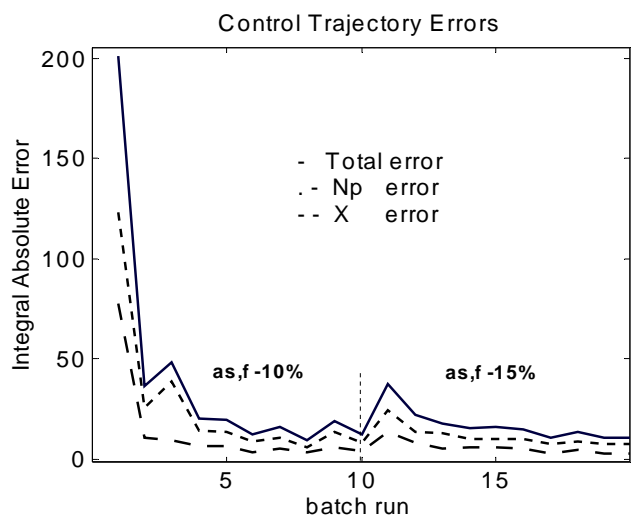

Fig. 5a. Trajectory control with adaptive algorithm. 

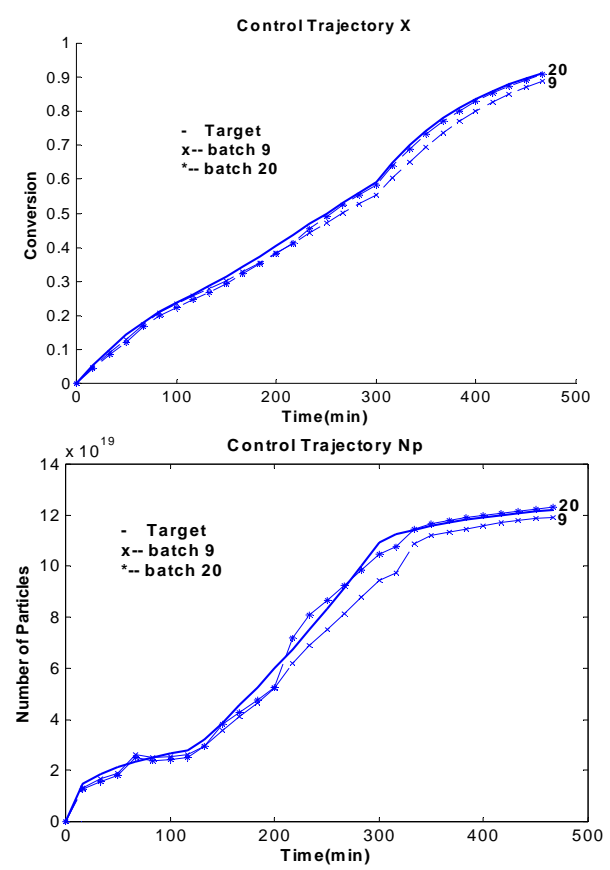

Fig. 5b. Trajectory control with adaptive algorithm.

\subsection{Case Study II. Control of PSD}

A detailed mechanistic model to simulate the PSD in emulsion polymerisation reactors was developed by Crowley et al. (2000). This simulator was used for data generation and control performance evaluation. For a description of the model and model parameters the reader is addressed to the original source, and for process conditions and recipes to Flores-Cerrillo and MacGregor (2002).

Two examples are presented for the PSD control in batch reactors when the process is subject to random, constant disturbances and model mismatch. In these examples, the control actions are shots of emulsifier and inhibitor at 30 and $150 \mathrm{~min}$ (total reaction time 380min). A PSD off-line measurement (sampled at 20min, with 10 min delay) is used to predict the end quality PSD.

\subsubsection{Example 1.}

In this example, PLS models are built from a data set consisting of 22 observations: 16 subject to random normal variations in as and $\mathrm{f}, 3$ with off-specification product and 3 in which mid-course corrections were performed. The obtained model has large error in the regression coefficients (nominal model).

To illustrate the performance of the learning control some results are presented. In Figure 6 is shown the evolution of the final PSD in the reduced and real space when a constant disturbance (batch to batch) affects the process. In this Figure, (o) is the control achieved using a PSD measurement. It can be seen in such Figure, that after 4 batches the control is almost perfect.

One of the main differences between batch-to-batch control and the proposed methodology is that the latter is an on-line scheme (The learning is performed off-line, but the control action is on-line). Therefore, the methodology can reject uncorrelated within batch disturbances. In Figure 7 is shown the control performance for uncorrelated disturbances using $\left(^{*}\right)$ Dp and (o) PSD measurements. It seems that the PSD is tracked on target in most of the batches. Learning between batches is also performed: There are disturbances that repeat after some batches; when they first appear, are farther from the target than when they reoccur.
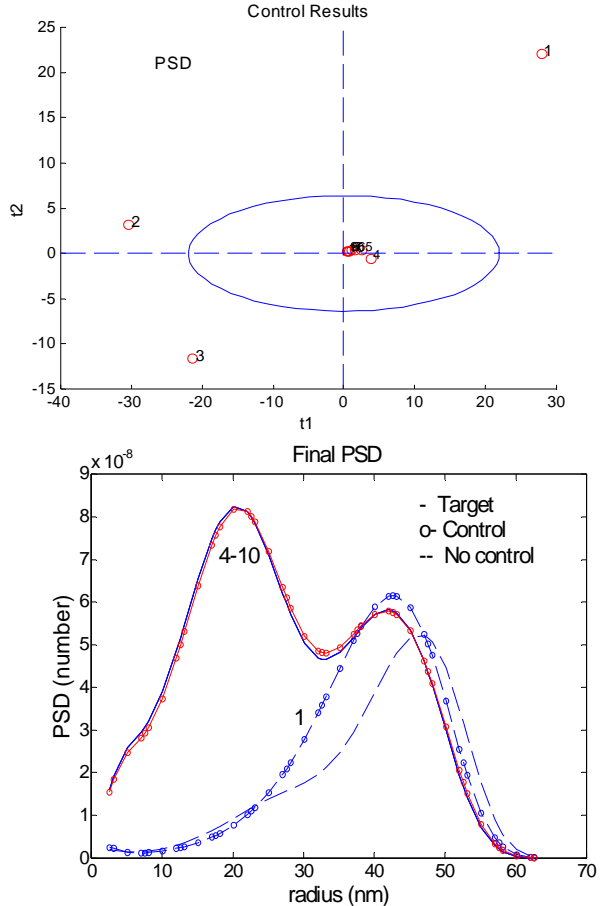

Fig. 6. PSD control with adaptive algorithm. $a_{s}=0.72 a_{s}^{*}, f=1.2 f^{*} .{ }^{*}$ nominal conditions
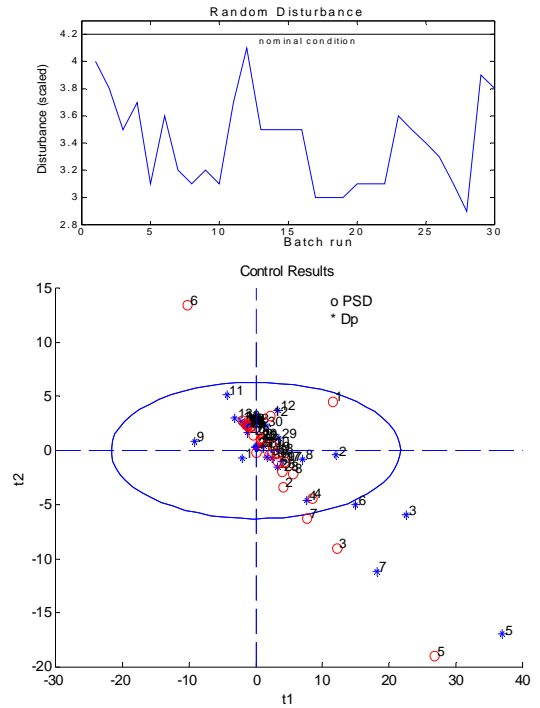

Fig. 7. PSD control with adaptive algorithm.

\subsubsection{Example 2}

Crowley et al. (2001), have used batch-to-batch optimisation for controlling the PSD when the process is subject to constant disturbances and model 
parameter mismatch. The prediction is performed using the theoretical model but correcting it with an updated PLS model. The PLS model relates the manipulated variables with the error from the theoretical model prediction and the measured distribution. This hybrid model is then used in a successive quadratic program to design surfactant and initiator input trajectories to drive the process on target. In the present work, the simpler learning online scheme is tested using only PLS models for online control. In Table 3 is shown the magnitude of the disturbances and those used by Crowley et al.

$\underline{\text { Table 3. Disturbance magnitudes }}$

\begin{tabular}{lcc} 
& $\begin{array}{c}\text { Example } \\
2^{*}\end{array}$ & $\begin{array}{c}\text { Crowley et al. } \\
(2001)\end{array}$ \\
\hline Critical micelle con. & 0.9 & 1.05 \\
Propagation rate & 0.95 & 0.95 \\
Particle coverage & 0.8 & 0.95 \\
Mon. part. droplet & 0.9 & 0.95 \\
Chain transfer rate & 1.05 & 1.05 \\
Diff. of mon. radical & 0.95 & 1.05 \\
Aq. termination & 0.95 & 1.05 \\
Initial surfactant & -- & $\sigma=0.00025$ \\
Particle coalescence & -- & $\sigma=0.0005$ \\
Coalescence Kernel & -- & $\mathrm{c}(1-\theta)(\mathrm{r} 3+\mathrm{r} ' 3)$ \\
Buffer concentration & 0.9 & -- \\
Diss. Constant & 1.2 & -- \\
*Multiplicative factors in nominal conditions.
\end{tabular}
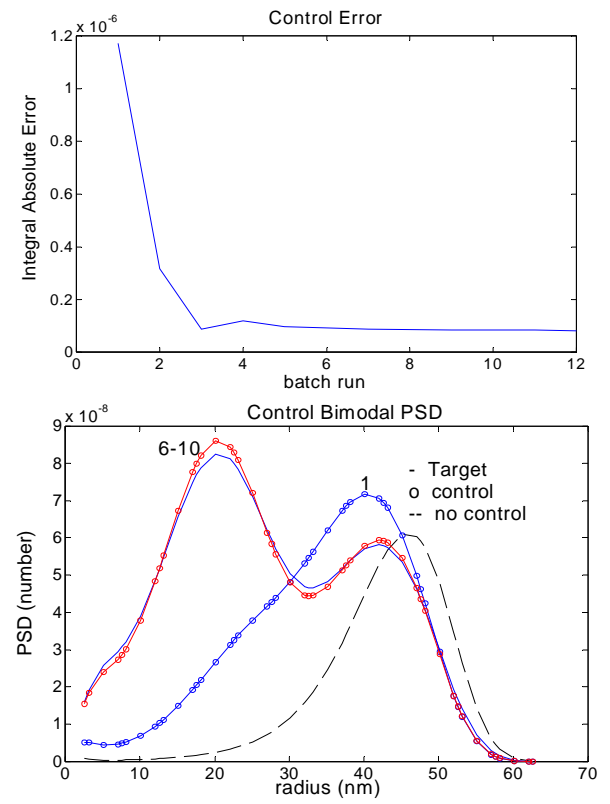

Fig. 8. PSD control with adaptive algorithm.

In Figure 8 is shown the control performance for this example, where it can be observed that the control error decreases after few batches and that the tracking is adequate.

\section{CONCLUSIONS}

An incremental learning methodology for the control of quality properties and trajectories in batch and semi-batch processes has been introduced. Learning algorithms were tested in lumped and distributed quality emulsion polymerisation processes subject to error initialisation, constant, correlated and random disturbances. This methodology is especially suitable for repetitive processes where the available information is limited, and for processes in which only few samples subject to analysis delay are available.

Important directions for this work include Monte Carlo simulations for testing the sensitivity of the control algorithms to different datasets and control including initial conditions (on-line-off-line scheme).

\section{ACKNOWLEDGMENTS}

J. Flores-Cerrillo thanks McMaster University and SEP-CONACYT for financial support and to Professor Doyle III and Crowley, T for their PSD emulsion polymerisation simulator.

\section{REFERENCES}

Crowley, T. J., E. S. Meadows, E. Kostoulas and F. J. Doyle III (2000). Control of Particle Size Distribution Described by a Population Balance Model of Semibatch Emulsion Polymerization. Journal of Process Control, 10, 419.

Crowley, T. J., C. A. Harrison and F. J. Doyle III (2001). Batch-to-Batch optimisation of PSD in emulsion polymerisation using a hybrid model. American Control Conference.

Flores-Cerrillo, J. and J.F. MacGregor (2002). Control of particle size distributions in emulsion semi-batch polymerization using mid-course correction policies. Accepted in Ind. Eng. Chem. Res.

Geladi, P. and B. R. Kowalski (1986). Partial LeastSquares Regression: A Tutorial. Anal. Chim. Acta, 185 (1), 1.

Kozub, D. J. (1989). Multivariate Control Design Robustness and Nonlinear Inferential Control for Semi Batch Polymerization Reactors. Ph. D. Thesis, Department of Chemical Engineering, McMaster University, Canada.

Lee, K. S. and J. H. Lee (2000). Convergence of Constrained Model-Based Predictive Control for Batch Processes. IEEE Transactions on Automatic Control, 45, 1928-1932.

Lee, J., K. S. Lee, J. H. Lee and P. Sunwon (2001). An on-line batch span minimization and quality control strategy for batch and semi-batch processes. Control Engineering Practice, 9, 901909.

Lynch, D. and C. Kiparissides (1981). Numerical Simulation of a Tubular Polymerization Reactor. J. of Applied polymer Science, 26, 1283-1293.

Russell, S. A., P. Kesavan, J. H. Lee and B. A. Ogunnaike (1998). Recursive Data-Based Prediction and Control of Batch Product Quality. AIChE Journal, 44, 2442-2458.

Yabuki, Y. and J.F. MacGregor (1997). Product Quality Control in Semibatch Reactors Using Midcourse Correction Policies. Ind. Eng. Chem. Res., 36, 1268-1275. 